\title{
Effects of hypertension and antihypertensive treatments on sulfatide levels in serum and its metabolism
}

Ran Guo, ${ }^{1}$ Xiao Hu, ${ }^{1}$ Yosuke Yamada, ${ }^{1,2}$ Makoto Harada, ${ }^{2}$ Takero Nakajima, ${ }^{1,3}$ Toshihide Kashihara, ${ }^{4}$ Mitsuhiko Yamada, ${ }^{4}$ Toshifumi Aoyama, ${ }^{1}$ Yuji Kamijo ${ }^{1,2, *}$

${ }^{1}$ Department of Metabolic Regulation, Shinshu University School of Medicine, 3-1-1 Asahi, Matsumoto,

Nagano, 390-8621, Japan

${ }^{2}$ Department of Nephrology, Shinshu University School of Medicine, 3-1-1 Asahi, Matsumoto, Nagano, 390-8621, Japan

${ }^{3}$ Research Center for Agricultural Food Industry, Shinshu University, Nagano, Nagano, 380-8553, Japan

${ }^{4}$ Department of Molecular Pharmacology, Shinshu University School of Medicine, 3-1-1 Asahi, Matsumoto,

Nagano, 390-8621, Japan

\section{* Correspondence:}

Yuji Kamijo, M.D., Ph.D.

Department of Nephrology, Shinshu University School of Medicine, 3-1-1 Asahi, Matsumoto 390-8621,

Japan

E-mail: yujibeat@shinshu-u.ac.jp

Tel: +81-263-37-2634

Fax: $+81-263-32-9412$

Running title: Effects of blood pressure alteration on serum sulfatides

Grants: This study was supported by Grants-in-Aid for Scientific Research (KAKENHI) in Japan (Grant Number: 18K08204). 


\section{Abstract}

Serum sulfatides are critical glycosphingolipids present in lipoproteins that work as modulators of thrombosis and hemostasis. Decreased serum sulfatide levels are suggested by our previous work to be related to cardiovascular disease (CVD). Hypertension, known to be an important risk factor for CVD, may affect serum sulfatide levels. However, how hypertension affects serum sulfatides directly and mechanistically is unknown.

To elucidate these possible mechanisms, we investigated changes in serum sulfatide levels and their metabolism using an established experimental model of hypertension that uses continuous infusion of angiotensin II (AngII) into mice. Furthermore, we also examined the effects of four different antihypertensive drugs (losartan, irbesartan, nifedipine and hydralazine) on serum sulfatide metabolism.

Serum levels of sulfatides were found to be decreased in groups in which only hypertension was induced (Angll only), while they were increased in groups with reduced blood pressure (antihypertensives only) and ameliorated to increasingly normal levels in groups with induced hypertension that were also treated (Angll+antihypertensives). Changes in serum sulfatides were strongly related to hepatic expression levels of cerebroside sulfotransferase (CST), which is a key enzyme involved in sulfatide synthesis. Furthermore, the current study suggests that the primary factors affecting CST expression are oxidative stress, peroxisome proliferator-activated receptor $\alpha$ activity and blood pressure itself.

This study demonstrates that hypertension significantly decreases levels of serum sulfatides by reducing hepatic CST expression via various effects mediated by Angll. Antihypertensive treatments can ameliorate abnormalities in serum sulfatide levels and may partially prevent hypertension related CVD by positively affecting sulfatide metabolism.

Keywords: blood pressure, serum sulfatides, CST, oxidative stress, PPAR $\alpha$ 


\section{Introduction}

Hypertension is a highly prevalent serious worldwide health problem. Approximately one third of the global adult population have hypertension, and nearly 13 million of these adults die each year from hypertension and its associated complications. ${ }^{1}$ Hypertension is the major risk factor for cardiovascular disease (CVD) due to its contribution to vascular tone, vascular remodeling and extracellular matrix formations. ${ }^{2}$ However, hypertension may affect other underlying mechanisms that may accelerate CVD development and warrant further investigation.

3-O-sulfogalactosylceramides (sulfatides), which are a class of glycosphingolipids composed of ceramide, galactose and sulfate, are known to exert multifunctional biological activities in various organs. ${ }^{3}$ Sulfatides exist in serum as major components of glycosphingolipids in serum lipoproteins. Serum sulfatides are produced mainly in the liver and are known to work as modulators of thrombosis and hemostasis. ${ }^{4,5}$ Sulfatides are also expressed on cell membranes of leukocytes, platelets and erythrocytes. These membranous sulfatides on blood cell surfaces accelerate coagulation and thrombosis, possibly through mechanisms that include by activating blood coagulation factor XII, by combining with annexin V, and by helping to stabilize platelet adhesion and aggregation. ${ }^{6,7}$ The previous study suggested that serum sulfatides can intervene in these interactions via membranous sulfatides, disturb the formation of fibrin and directly inhibit thrombin activity. ${ }^{6,8}$ Therefore, abnormalities in serum sulfatide levels may affect the pathogenesis of CVD by exhibiting anticoagulant properties. Indeed, our past clinical study investigating hemodialysis (HD) patients, a very high-risk group for CVD, suggested this relationship. This study demonstrated that CVD incidence is significantly increased in HD patients with lower serum sulfatide levels, compared with those with higher levels. These results suggested that the reduction of serum sulfatide levels might induce development of CVD and that preventing abnormalities in serum sulfatide levels might 
useful in CVD prevention. ${ }^{9}$ Understanding the underlying mechanism is essential for establishing an intervention method. However, the factors influencing serum sulfatide metabolism have not been thoroughly elucidated to date.

Several earlier experimental studies suggest that the reduction of serum sulfatide levels in pathological conditions, such as acute kidney injury, ${ }^{10}$ was caused mainly by a decreased hepatic expression of cerebroside sulfotransferase (CST), a key enzyme for sulfatide synthesis and that CST expression was decreased by oxidative stress (OS) and increased by activation of peroxisome proliferator-activated receptor $\alpha(\operatorname{PPAR} \alpha),{ }^{5,11}$ a common transcription factor. We hypothesized that hypertension, which is closely related to CVD pathogenesis, may potentially influence serum sulfatide levels.

To investigate this hypothesis and the associated underlying mechanism, we examined changes of serum sulfatide levels and the associated mechanism using an established experimental model of hypertension where a mouse is given a continuous infusion of angiotensin II (AngII). To eliminate what possible direct effects AngII may have on sulfatide regulation, we used two kinds of angiotensin II receptor blockers (ARBs): a typical ARB (losartan), and a unique ARB (irbesartan) known to activate PPAR $\alpha^{12,13}$ We also wanted to evaluate how serum sulfatide levels changed in conditions where blood pressure was lowered. A representative calcium channel blocker (CCB) (nifedipine) and a direct smooth muscle vasodilator (hydralazine) were chosen, that have minimal effects on sphingolipid metabolism. ${ }^{14}$ Four different antihypertensive drugs were administered into sham surgery mice and mice with induced hypertension through Angll infusion, respectively. Changes in serum sulfatide levels and the underlying mechanism for these changes were subsequently investigated. 


\section{Methods}

\section{Animal experiments}

All animal experiments were conducted in accordance with animal study protocols outlined in the Guide for the Care and Use of Laboratory Animals prepared by the National Academy of Sciences and carried out in accordance with the Regulations for Animal Experimentation of Shinshu University. The animal protocol was approved by the Committee for Animal Experiments of Shinshu University (Approval Number 290006). Ten-week-old male C57BL/6J mice weighting 20-25 g were maintained under controlled condition $\left(25^{\circ} \mathrm{C} ; 12\right.$-h light/dark cycle) with tap water and randomly assigned into four groups: 1$)$ Control (Con) group $(n=10), 2)$ hypertension (HT) groups that included a low dose continuous infusion of angiotensin II (Angll; SIGMA-Aldrich, MO, USA) into mice $(0.2 \mathrm{mg} / \mathrm{kg} /$ day; $\mathrm{n=5}$ ) or a high dose continuous infusion into mice $(0.5 \mathrm{mg} / \mathrm{kg} /$ day; $\mathrm{n}=5), 3)$ low blood pressure (LBP) groups that included mice that were given losartan (Los;30 mg/kg/day; LKT Laboratories, MN, USA), mice that were given irbesartan (Irbe;30 mg/kg/day; Sumitomo Dainippon Pharma, Tokyo, Japan), mice that were given nifedipine (Nif;24 $\mathrm{mg} / \mathrm{kg} /$ day; NIHON GENERIC, Tokyo, Japan) or mice that were given hydralazine (Hyd;5 mg/kg/day; SUN PHARMA, Tokyo, Japan), n=5 for each group, respectively, 4) hypertension treatment (HT-T) groups; high dose Angll infusion was given to mice along with an antihypertensive medication; these groups were Angll+Los, AngII+Irbe, AngII+Nif or Angll+Hyd, $\mathrm{n}=5$ for each group, respectively. HT and HT-T mice were anesthetized by a mixture of medetomidine hydrochloride, butorphanol tartrate and midazolam, and osmotic minipumps (model 1004 ALZET/DURECT Corporation, Cupertino, CA, USA) were implanted subcutaneously into the interscapular region to allow continuous infusion of an Angll-mixed saline solution. ${ }^{15}$ Meanwhile, Con and LBP mice received sham surgery to eliminate impacts of surgery might have had. LBP and HT-T mice were maintained on a standard rodent diet mixed with antihypertensive drugs, whereas Con and HT were only given a standard diet. Each group's food intake was measured every 
fourth day for the duration of the experiments to verify that the drug dose was constant. The doses of antihypertensive drugs used in present study were adjusted and set by preliminary experiments (Supplementary preliminary experiment methods and supplemental Fig. 1), where various doses of antihypertensive drugs were administered to obtain a very similar reduction of systolic blood pressure (SBP). All mice were sacrificed under anesthesia at day 28 , and serum and livers were collected from each mouse and stored at $-80^{\circ} \mathrm{C}$ until they were analyzed.

\section{Blood Pressure Monitoring}

SBP was measured in conscious mice using a non-invasive tail-cuff apparatus (BP-98A, Softron Co., Tokyo, Japan) prior to the experiment and every 7 days in experiments with extended durations. At each time point, over 20 measurements were recorded and averaged.

\section{Quantitation and qualitative analyses of sulfatides}

Sulfatides were extracted from serum and liver-homogenate of each mouse with n-hexane/isopropanol solution $(3: 2 \mathrm{v} / \mathrm{v})$, then these extracts were converted to lysosulfatides (LS; sulfatides without fatty acids) by saponification with methanolic sodium hydroxide as described previously. ${ }^{16}$ The resulting LS samples were purified through Mono-tip C18 cartridges (GL Sciences, Tokyo, Japan). Following the addition of the internal standard, N-acetylated LS-sphinganine (LS-d 18:0 NAC), the samples were analyzed by matrix-assisted laser desorption ionization-time of flight mass spectrometry (MALDI-TOF MS) using a TOF/TOF 5800 system (AB Sciex, Framingham, MA, USA) in negative ionization mode. A two-point external calibration was performed with LS-d18:0 NAc $\left([\mathrm{M}-\mathrm{H}]^{-}=584.310\right)$ and LS-(4E)-sphinganine (LS-d18:1) $\left([\mathrm{M}-\mathrm{H}]^{-}=540.284\right)$. The following seven molecular species of LS were detected based on the differences in their sphingoid base structure: LS-sphingadienine (LS-d18:2), LS-d18:1, LS-sphinganine (LS-d18:0), LS-phytosphingosine (LS-t18:0), LS-(4E)-icosasphingenine (LS-d20:1), 
LS-icosasphingenine (LS-d20:0), and LS-4D-hydroxyicosasphingenine (LS-t20:0). Total levels of sulfatides were calculated as the sum of the levels of these seven LS species. ${ }^{17}$

\section{Analysis of mRNA}

Liver total RNA was extracted using an RNeasy Mini Kit (QIAGEN, Hilden, Germany). One microgram of total RNA was reverse-transcribed using a PrimeScript RT Reagent kit (Takara Bio, Otsu, Japan). mRNA levels were measured through quantitative real-time polymerase chain reactions (PCR) using a SYBR Premix EX Taq II (Takara Bio) on a StepOnePlus Real-Time PCR System (Applied Biosystems, Foster, CA, USA). Gene-specific primers were designed by Primer Express software (Applied Biosystems) as shown in Supplementary Table 1. mRNA encoding for glyceraldehyde-3-phosphate dehydrogenase (Gapdh) was used as an endogenous control to normalize the samples using the $\Delta \Delta C T$ method and later expressed as fold changes relative to that of mice in the Con group.

\section{Immunoblot Analysis}

Liver cytosolic and nuclear fractions were extracted from each mouse using Cytoplasmic Extraction, and NE-PER Nuclear Reagents (Thermo Fisher Scientific, Rockford, IL, USA). Protein concentrations were determined colorimetrically with a BCA Protein Assay kit (Thermo Fisher Scientific). Lysates of nuclear and cytosolic proteins $(30 \mu \mathrm{g})$ were subjected to $10 \%$ sodium dodecyl sulfate-polyacrylamide gel electrophoresis (SDS-PAGE). After electrophoresis, proteins were transferred onto a nitrocellulose membrane (Amersham Hybond-P; GE Health Care, Little Chalfont, UK). These membranes were blocked for 1 hour in 1\% nonfat dry milk in Tris-buffered saline and later incubated overnight with the respective primary antibody at $4^{\circ} \mathrm{C}$. The membranes were washed four times and subsequently incubated with peroxidase-conjugated secondary antibodies (Jackson ImmunoResearch Laboratories, West Grove, PA, 
USA) and treated with ECL Prime Western Blotting Reagents (GE Health). Primary antibodies for long-chain acyl-coenzyme A synthase (LACS), peroxisomal thiolase (PT) and catalase were described previously $^{18}$ and others were purchased commercially: antibodies against $\beta$-actin, TATA box-binding protein (TBP) and serine palmitoyl-CoA transferase (SPT) were from Abcam (Cambridge, UK), antibody against cerebroside sulfotransferase (CST) was from Abnova (Taipei, Taiwan), anti-arysulfatase A (ARSA) was obtained from Everest Biotech (Oxfordshire, UK), anti-glycolipid transfer protein (GLTP) antibody was obtained from Proteintech (Chicago, IL, USA), and the following primary antibodies were purchased from Santa Cruz Biotechnology (Dallas, CA, USA): ceramide galactosyltransferase (CGT), galactosylceramidase (GALC), neutrophil cytosolic factor 1 (NCF1), NADPH oxidase 2 (NOX2), superoxide dismutase 1 (SOD1), glutathione peroxidase 1 (GPX1) and peroxisome proliferator-activated receptor $\alpha$ (PPAR $\alpha$ ). The positions of protein bands were determined by co-electrophoresing molecular weight standards (Bio-Rad, Hercules, CA, USA), and the bands of actin and TBP were used as loading controls. Immunoblotting was repeated at least twice for each protein, and the band intensity was measured densitometrically through normalization to the loading control and subsequently expressed as fold changes relative to that of mice in the Con group.

\section{Measurement of lipid peroxides}

The concentration of malondialdehyde (MDA) in the liver was measured using a MDA-586 kit (OXIS International, Beverly Hills, CA, USA).

\section{Biochemical analysis}

Serum alanine aminotransferase (ALT) and aspartate aminotransferase (AST) levels were measured using enzyme assay kits (Wako Pure Chemical Industries, Ltd., Osaka, Japan). 


\section{Statistical Analysis}

Results are expressed as the mean \pm SEM. Statistical analysis was performed using one-way analysis of variance (ANOVA), followed by Fisher's PLSD post-hoc test. A probability value less than 0.05 was considered to be statistically significant. 


\section{Results}

\section{Continuous Angll infusion increased SBP, whereas antihypertensive treatments ameliorated SBP to normal levels.}

All mice received osmotic minipump implantation surgery or a sham surgery, where no mice died during operation, and systemic conditions indicated all of them had recovered well. For 28-day experiment durations, there were no symptoms of abnormal behavior or eating disorders in these mice.

SBP was measured and analyzed as shown in Fig. 1. A continuous high dose infusion of Angll gradually and significantly increased SBP at the seventh day after initiating infusion compared to before treatment was initiated. Although the SBP of Angll low dose infusion mice was also raised significantly at the fourteenth day after treatment had begun, the elevation of SBP was mild and did not reach our requirements for a hypertension model. Thus we chose the high dose of Angll infusion as a reliable hypertension model, and we used this experimental condition in our experiments with antihypertensive drugs. Consistent with our preliminary experiment results (Supplementary preliminary experiment methods and supplemental Fig. 1), SBP was similarly and remarkably reduced in LBP groups with suitable doses of the four antihypertensive drugs at the seventh day after infusion had begun until the experiment finished. As expected, each antihypertensive treatment in each HT-T group ameliorated the increased SBP observed in mice where Angll was infused at a high dose, and SBP was approximately reduced to the SBP level observed at the start of each experiment.

Other basic indices in mice aside from SBP, which included body weight, liver weight, kidney weight, heart weight, food intake, and representative liver injury markers ALT and AST did not differ significantly among groups (Supplementary Table 2).

Serum sulfatide levels were reduced in HT groups but were increased by antihypertensive treatments in LBP and HT-T groups. 
To investigate whether hypertension affects serum sulfatide levels, we measured serum sulfatide levels using samples from each experimental mouse. As shown in Fig. 2A, serum sulfatide levels were significantly decreased in HT groups in an Angll dose-dependent manner. In LBP groups, all antihypertensive treatments increased serum sulfatide levels compared to the control group, and among them, the ARB treatments exhibited the most prominent effects, particularly in Irbe-treated mice. Nif and Hyd had similar effects on elevating serum sulfatide levels, but the effects were milder than those observed with ARBs. These antihypertensive treatments could ameliorate the decline of serum sulfatide levels in mice that were continuously infused with a high dose of Angll. In HT-T groups, serum sulfatide levels in AngII+Irbe mice were almost recovered to control levels. Since our previous study reported that serum sulfatides are secreted from the liver, we also measured liver sulfatides. ${ }^{4,5}$ Changes in liver sulfatides mirrored those observed in serum (Fig. 2A). Meanwhile, the sphingoid compositions of serum sulfatides did not differ among groups and were approximately identical to that observed in hepatic sulfatides (Fig. 2B). This finding implies that neither Angll infusion nor antihypertensive treatments affected serum and liver sulfatide compositions. Overall, these results indicate that hypertension decreased serum and liver sulfatide levels, and that antihypertensive treatments can ameliorate those reductions.

\section{Serum sulfatide levels were mainly related to the hepatic expression of CST.}

To clarify the mechanism causing the serum sulfatide changes mentioned above, hepatic mRNA expression of sulfatide-metabolizing enzymes were measured. mRNA expression of CST was obviously decreased in the HT groups in an Angll dose-dependent manner (Fig. 3A). Inversely, mRNA expression of 
CST in the LBP groups was significantly increased compared to what was observed in controls, especially in mice treated with ARBs. Irbe-treated mice showed the greatest altered CST mRNA expression of all the LBP groups. CST expressions in Nif and Hyd mice were also identically increased compared to what is observed in control mice; however, the increase observed was lower than that observed in mice treated with ARBs. In HT-T groups, treatment with ARBs significantly ameliorated the reduced expression of CST in mice continuously infused with a high dose of Angll. The recovered levels of CST in Angll+Los mice were still less than levels that were observed in control mice; however Angll+Irbe mice nearly reached control levels. CST expressions in Angll+Nif and Angll+Hyd mice tended to show increased levels compared to those observed in mice that were only continuously infused with a high dose of Angll; however, the observed recovery was mild. Immunoblot analysis also confirmed that trends of CST hepatic protein expression in these mice were almost identical to mirrored changes in mRNA expression (Fig. 3B). We also assessed other sulfatide-metabolic enzymes involved CGT, responsible for synthesis of galactosylceramides; SPTLC2, a rate-limiting enzyme in the synthesis of ceramides/sphingolipids that include sulfatides; ARSA, a main enzyme affecting sulfatide degradation; GALC, an enzyme causing degradation of galactosylceramides to ceramides; and GLTP, proteins relating to sphingolipid transfer. mRNA expression and protein levels were not observed to be different in these groups (Fig. 3A and Supplementary Fig. 2). In brief, these results suggested that the hepatic expression of CST is primarily responsible for changes in levels of serum sulfatides.

Hepatic OS level was remarkably increased in HT groups; ARB treatments improved the elevation of hepatic OS but neither nifedipine nor hydralazine caused improvement.

As previously reported, increased OS could reduce hepatic CST expression, thereby inducing 
reduction in serum sulfatide levels. ${ }^{11,19}$ Hence, we measured the level of malondialdehyde (MDA) as a marker for hepatic OS, with the results shown in Fig. 4A. MDA levels were significantly increased in the HT groups when compared to controls. MDA levels were significantly decreased in mice treated with ARBs in the LBP groups compared to controls, but no changes were observed in Nif- and Hyd-treated mice in these LBP groups. In HT-T groups, MDA levels were significantly decreased in AngII+ARBs treated mice compared to mice with a continuous infusion of a high dose of Angll, and reached similar levels to those observed in the control group. However, this reduction in levels of OS was not found in Angll+Nif or Angll+Hyd mice, and MDA levels of these two groups were identical to those in mice receiving a continuous infusion of a high dose Angll.

We assessed hepatic enzymes involved in regulating the generation and elimination of reactive oxygen species (ROS) to further describe the mechanism proposed above. mRNA expression of catalase, which is related to removing ROS, was increased only in Irbe mice in the LBP and HT-T groups. However, mRNA levels of other ROS elimination enzymes, SOD1 and GPX1, did not differ among the groups (Fig. 4B). NOX2 and NCF1, typical enzymes associated with ROS generation, were assessed. Hepatic mRNA expression of NOX2 was increased in the high dose HT group compared to controls and significantly decreased in the Angll+ARBs HT-T group compared to the high dose HT group. The NOX2 suppressive effect was not observed in Angll+Nif or Angll+Hyd HT-T groups. mRNA expression of NCF1 was identical in all groups. Another enzyme associated with ROS generation, ACOX1, was also measured. ACOX1 expression tended to increase in the high dose HT group as compared to controls $(P=0.054)$, but no changes found in any of the LBP groups. Moreover, these four antihypertensive treatments could not suppress the elevation of ACOX1 expressions induced by Angll infusion. Assessing protein levels rendered identical findings (Fig. 4C and Supplementary Fig. 2). 


\section{Irbesartan treatment increased the hepatic expression of PPAR $\alpha$ and its target enzymes.}

Another study has demonstrated that PPAR $\alpha$ activation could increase hepatic CST expression in mice

apart from increased OS affecting hepatic CST expression. ${ }^{5}$ Therefore, hepatic PPAR $\alpha$ and its representative target enzymes, including LACS and PT, were evaluated. mRNA expression of Ppara, Lacs and Pt were all only significantly increased in Irbe-treated mice in both the LBP groups and the HT-T groups. All other treatment groups showed no differences compared to controls (Fig. 5A). Irbe-specific PPAR $\alpha$-activation was also observed at the protein level (Fig. 5B). In agreement with the previous study, these results indicate that irbesartan activates hepatic PPAR $\alpha$. This Irbe-specific PPAR $\alpha$-activation might enhance hepatic expression of CST. 


\section{Discussion}

There has not been research focused on the relationship between hypertension and levels of serum sulfatide. The present study reveals that serum and liver sulfatide levels were remarkably decreased in a hypertension model induced by continuous infusion of Angll in a manner depending on the overall dose of AngIl. Administration of different antihypertensive drugs can significantly ameliorate reductions of serum and liver sulfatide levels, with the amelioration effects being dependent on the various mechanisms of each antihypertensive drug. The change in sulfatide metabolism was most likely associated with altered hepatic CST expression. Additionally, under the current experimental conditions, we found that hepatic CST expression influenced factors were primarily attributed to enhancing OS, activating PPAR $\alpha$ and lowering blood pressure itself. Proposed associations of these mechanisms are depicted in Fig.6.

There is accumulating evidence verifying the interactions between OS and hypertension. Vascular ROS production is previously reported to be increased in a hypertensive situation. ${ }^{20,21}$ Furthermore, the link between vascular OS and hypertension is known to be bidirectional: OS promotes the development of hypertension and contributes to hypertension-induced pathologies. ${ }^{22}$ Administrations of antioxidants, such as tempol, or membrane-targeted forms of SOD are reported to possibly lower blood pressure, similar to an antihypertensive drug. ${ }^{23,}{ }^{24}$ In the current study, nifedipine and hydralazine treatments successfully lowered blood pressure but not the levels of OS, whereas ARBs were shown to improve both hypertension and OS in the same group. Moreover, there are few reports indicating an anti-oxidative ability in non-RAS inhibitor types of antihypertensives. These findings suggest that the related mechanisms between OS and hypertension would be mainly due to RAS activation. It is well accepted that Angll is a potent activator of NADPH oxidase in the cardiovascular system and contributes to ROS production. ${ }^{25}$ AngIl also enhances the levels of OS in other organs apart from those in the cardiovascular system, such as 
the kidneys and the liver. $^{26,27}$ A previous study demonstrated that enhancement of os induced the suppression of hepatic CST expression in a murine model of chronic ethanol consumption. ${ }^{4}$ In clinical research, improvement of OS recovers reduced serum sulfatide levels in kidney transplantation patients. ${ }^{11,}$ ${ }^{28}$ Although there is no unambiguous evidence to clarify how improving OS affect hepatic CST expression, OS is considered as a key mechanistic mediator of levels of sulfatides. The current findings demonstrated that Angll-induced HT significantly increased the levels of OS and reduced hepatic CST expression. Furthermore, treatments with ARBs, losartan and irbesartan all completely attenuated increased hepatic levels of OS induced by Angll infusion, and the hepatic expression of CST in these mice was prominently recovered. These findings suggest the close relationship between RAS activation-induced OS and CST expression. However, although non-ARB antihypertensives Nif and Hyd scarcely exert anti-oxidative effects, the CST and serum sulfatide levels of Nif- or Hyd-treated mice in LBP groups were mildly increased. Moreover, reduced CST and serum sulfatide levels in Nif- or Hyd-treated mice in HT-T groups recovered partially. These findings suggest that factors other than OS may influence sulfatide metabolism.

In recent years, we have studied interactions between PPAR $\alpha$ and sulfatides, as well as PPAR $\alpha$ and ARBs, and made several important discoveries. Our past studies indicated that PPAR $\alpha$ activation increased CST gene expression and enhanced sulfatide synthesis in several murine organs, which included the liver, suggesting that CST is a gene targeted through PPAR $\alpha$ activation. ${ }^{29}$ Recently, we reported that irbesartan, a unique angiotensin II receptor blocker, could activate renal PPAR $\alpha$ in mice experiencing protein-overload nephropathy. ${ }^{12}$ Other studies from other research groups also demonstrated that irbesartan can up-regulate PPAR $\alpha$-activated genes and genes related to fatty acid oxidation in the livers of obese Koletsky rats. ${ }^{13}$ These findings suggested that irbesartan is a reliable PPAR $\alpha$ agonist. In the present study, hepatic expression of PPAR $\alpha$ and its representative target enzymes LACS and PT were increased through 
an irbesartan-specific effect, which is consistent with past results. In the current study, the degree of increased CST expression in mice treated with irbesartan was much higher than what was observed in mice treated with other antihypertensives in the LBP groups and HT-T groups. This obvious effect of irbesartan causing increased CST might de due to its ability to activate PPAR $\alpha$. Furthermore, PPAR $\alpha$ activation is known to exert antioxidant effects by inducting ROS eliminating enzymes, such as catalase. ${ }^{18,}$ ${ }^{30}$ These PPAR $\alpha$-mediated anti-oxidative effects would reduce OS and may contribute to the increase in sulfatide levels. The double-effects of PPAR $\alpha$ activation by direct induction of CST and induction of CST mediated by anti-oxidation effects increased sulfatide levels intensively in Irbe-treated mice, adding to the common classic ARB effects of RAS suppression.

Interestingly, although no OS amelioration or PPAR $\alpha$ activation was observed in either Nif- or Hyd-treated mice, hepatic CST expression and sulfatide levels in these mice were significantly and similarly improved. Mice blood pressures were downregulated by four different antihypertensives similarly in LBP groups (SBP $100 \pm 2.7$ vs $110 \pm 3.7$ control) and effectively improved to control levels in HT-T groups (SBP $110 \pm 4.8$ vs $110 \pm 3.7$ control). Since these four drugs decreased blood pressure to the same extent via different mechanisms, it is possible that CST expression and sulfatide levels are influenced by blood pressure alterations directly, which may become an important breakthrough point. Previous studies indicated that losartan and hydralazine decreased vascular ceramide levels and plasma ceramide levels in spontaneously hypertensive rats (SHR) possibly due to lowered blood pressure, although the exact mechanism is currently unknown. ${ }^{31}$ Since sulfatides are derivative of ceramides, the reduction of ceramide levels might reflect the increase of sulfatide synthesis in conditions where blood pressure is decreased. Contrastingly, lowered blood pressure by antihypertensive treatments including ARB, CCB and hydralazine, is reported to possibly improve insulin resistance (IR) and suppress inflammatory cytokines 
such as tumor necrosis factor (TNF)- $\alpha$ and interleukins (IL)-6. ${ }^{32-35}$ An earlier study reported that pharmacological intervention in glycosphingolipid biosynthesis may modulate IR in animal models. ${ }^{36}$ Furthermore, serum sulfatide levels in Type 2 diabetes mellitus patients (where almost all these patients are in an IR state), were significantly decreased. ${ }^{37}$ In addition, TNF- $\alpha$ and IL-6 are reported to be critical factors for inducing IR, and expressions of these inflammatory cytokines are reduced by sulfatides. ${ }^{38,39}$ These findings suggest that affected sulfatide metabolism and HT induced IR, and inflammatory cytokines may be closely linked. Therefore, improving hypertension may change sulfatide metabolism through reducing IR and inflammatory cytokines; however, this hypothesis is not confirmed in the current study, and future studies will be necessary.

Although the present study provides the first suggestion that lowering blood pressure could increase serum sulfatide levels, there are several limitations that must be noted. First, we used a tail cuff method to measure blood pressure. This method is generally associated with wider blood pressure variation than continuous blood pressure monitoring. Therefore, it is possible that we could not detect significant differences in blood pressure among the groups because of variability in measurements. Second, the current study suggests that reversing the reduction of serum sulfatide levels induced by hypertension contributed to normalization of serum sulfatide function. However, it is unclear from the present study whether very high serum sulfatide levels exert beneficial effects. Sulfatides are reported to have both anticoagulant and procoagulant activities. It was reported that when injected into mice with heavily damaged blood vessel walls or when continuously infused into mice through plastic cannulae, sulfatides enhanced thrombosis and blood coagulation. ${ }^{8}$ Under in vitro and ex vivo experimental conditions using initially activated platelets, sulfatide-P-selectin interactions are reported to increase platelets activation and aggregation. ${ }^{40}$ Additionally, Li et al. recently reported that serum sulfatide levels are abnormally high 
in ST-segment elevation myocardial infarction (STEMI) patients and that this is positively correlated to STEMI complications. ${ }^{41}$ We believe that maintaining serum sulfatides at a normal level is clinically important, but further study is necessary to determine what that level is. Third, we estimated serum sulfatide levels in a single condition of lowering blood pressure; therefore, the accurate correlation curve between continuous blood pressure alterations and serum sulfatide levels could not be determined. Lastly, we cannot distinguish whether blood pressure elevation or increased OS levels decreased sulfatide levels in the current study, and more thorough experiments will be needed in the future.

In summary, this study revealed that serum sulfatides decreased in an established experimental model of hypertension by using a continuous infusion of Angll in mice, and can be ameliorated using antihypertensive treatments. The mechanism of serum sulfatide alteration is primarily related to changes in hepatic CST expression. In the present study, CST expression was influenced by multiple factors, which included oxidation stress, peroxisome proliferator-activated receptor $\alpha$ activation and blood pressure itself. Importantly, this relationship between hypertension and serum sulfatides may provide a potential therapeutic direction for the future treatment of hypertension related to CVD.

\section{Acknowledgments}

None.

\section{Conflict of Interest}

The authors have declared that no conflict of interest exists. 


\section{References}

1. Lawes CMM, Hoorn SV, Rodgers A. Global burden of blood-pressure-related disease, 2001. The Lancet. 2008; 371: 1513-1518.

2. Intengan HD, Schiffrin EL. Vascular remodeling in hypertension: Roles of apoptosis, inflammation, and fibrosis. Hypertension. 2001; 38: 581-587.

3. Takahashi T, Suzuki T. Role of sulfatide in normal and pathological cells and tissues. J. Lipid Res. 2012; 53: $1437-1450$.

4. Kanbe H, Kamijo Y, Nakajima T, Tanaka N, Sugiyama E, Wang L, Fang Z-Z, Hara A, Gonzalez FJ, Aoyama T. Chronic ethanol consumption decreases serum sulfatide levels by suppressing hepatic cerebroside sulfotransferase expression in mice. Arch. Toxicol. 2014; 88: 367-379.

5. Kimura T, Nakajima T, Kamijo Y, Tanaka N, Wang L, Hara A, Sugiyama E, Tanaka E, Gonzalez FJ, Aoyama T. Hepatic cerebroside sulfotransferase is induced by ppara activation in mice. PPAR research. 2012; 2012:

6. Merten M, Thiagarajan P. Role for sulfatides in platelet aggregation. Circulation. 2001; 104: 2955-2960.

7. Ida M, Satoh A, Matsumoto I, Kojima-Aikawa K. Human annexin v binds to sulfatide: Contribution to regulation of blood coagulation. J. Biochem. 2004; 135: 583-588.

8. Kyogashima M. The role of sulfatide in thrombogenesis and haemostasis. Arch. Biochem. Biophys. 2004; 426: 157-162.

9. Hu R, Li G, Kamijo Y, Aoyama T, Nakajima T, Inoue T, Node K, Kannagi R, Kyogashima M, Hara A. Serum sulfatides as a novel biomarker for cardiovascular disease in patients with end-stage renal failure. Glycoconj. J. 2007; 24: 565-571.

10. Zhang X, Nakajima T, Kamijo Y, Li G, Hu R, Kannagi R, Kyogashima M, Aoyama T, Hara A. Acute kidney injury induced by protein-overload nephropathy down-regulates gene expression of hepatic cerebroside sulfotransferase in mice, resulting in reduction of liver and serum sulfatides. Biochem. Biophys. Res. Commun. 2009; 390: 1382-1388.

11. Kamijo Y, Wang L, Matsumoto A, Nakajima T, Hashimoto K, Higuchi M, Kyogashima M, Aoyama T, Hara A. Long-term improvement of oxidative stress via kidney transplantation ameliorates serum sulfatide levels. Clin. Exp. Nephrol. 2012; 16: 959-967.

12. Harada M, Kamijo Y, Nakajima T, Hashimoto K, Yamada Y, Shimojo H, Gonzalez FJ, Aoyama T. Peroxisome proliferator-activated receptor $\alpha$-dependent renoprotection of murine kidney by irbesartan. Clin. Sci. 2016; 130: 1969-1981.

13. Rong X, Li Y, Ebihara K, Zhao M, Kusakabe T, Tomita T, Murray M, Nakao K. Irbesartan treatment up regulates hepatic expression of ppar a and its target genes in obese koletsky (fak/fak) rats: A link to amelioration of hypertriglyceridaemia. Br. J. Pharmacol. 2010; 160: 1796-1807.

14. Zisaki A, Miskovic L, Hatzimanikatis V. Antihypertensive drugs metabolism: An update to pharmacokinetic profiles and computational approaches. Curr. Pharm. Des. 2015; 21: 806-822.

15. Kashihara T, Hirose M, Shimojo H, Nakada T, Gomi S, Hongo M, Yamada M. B2-adrenergic and m2-muscarinic receptors decrease basal t-tubular I-type ca2+ channel activity and suppress ventricular contractility in heart failure. Eur. J. Pharmacol. 2014; 724: 122-131.

16. Hara A, Radin NS. Lipid extraction of tissues with a low-toxicity solvent. Anal. Biochem. 1978; 90: 420-426.

17. Li G, Hu R, Kamijo Y, Nakajima T, Aoyama T, Inoue T, Node K, Kannagi R, Kyogashima M, Hara A. Establishment of a quantitative, qualitative, and high-throughput analysis of sulfatides from small 
amounts of sera by matrix-assisted laser desorption ionization-time of flight mass spectrometry. Anal. Biochem. 2007; 362: 1-7.

18. Aoyama T, Peters JM, Iritani N, Nakajima T, Furihata K, Hashimoto T, Gonzalez FJ. Altered constitutive expression of fatty acid-metabolizing enzymes in mice lacking the peroxisome proliferator-activated receptor $\alpha$ (ppar $\alpha)$. J. Biol. Chem. 1998; 273: 5678-5684.

19. Yuzhe H, Kamijo Y, Hashimoto K, Harada M, Kanno T, Sugiyama E, Kyogashima M, Oguchi T, Nakajima T, Kanno Y. Serum sulfatide abnormality is associated with increased oxidative stress in hemodialysis patients. Hemodialysis International. 2015; 19: 429-438.

20. Chabrashvili T, Tojo A, Onozato ML, Kitiyakara C, Quinn MT, Fujita T, Welch WJ, Wilcox CS. Expression and cellular localization of classic nadph oxidase subunits in the spontaneously hypertensive rat kidney. Hypertension. 2002; 39: 269-274.

21. Wilcox CS. Oxidative stress and nitric oxide deficiency in the kidney: A critical link to hypertension? American Journal of Physiology-Regulatory, Integrative and Comparative Physiology. 2005; 289 : R913-R935.

22. Harrison DG, Gongora MC. Oxidative stress and hypertension. Medical Clinics. 2009; 93: 621-635.

23. Zimmerman MC, Dunlay RP, Lazartigues E, Zhang Y, Sharma RV, Engelhardt JF, Davisson RL. Requirement for rac1-dependent nadph oxidase in the cardiovascular and dipsogenic actions of angiotensin ii in the brain. Circ. Res. 2004; 95: 532-539.

24. Zimmerman MC, Lazartigues E, Lang JA, Sinnayah P, Ahmad IM, Spitz DR, Davisson RL. Superoxide mediates the actions of angiotensin ii in the central nervous system. Circ. Res. 2002; 91: 1038-1045.

25. Landmesser U, Cai H, Dikalov S, McCann L, Hwang J, Jo H, Holland SM, Harrison DG. Role of p47phox in vascular oxidative stress and hypertension caused by angiotensin ii. Hypertension. 2002; 40: 511-515.

26. Zhong J, Guo D, Chen CB, Wang W, Schuster M, Loibner H, Penninger JM, Scholey JW, Kassiri Z, Oudit GY. Prevention of angiotensin ii-mediated renal oxidative stress, inflammation, and fibrosis by angiotensin-converting enzyme 2. Hypertension. 2010: HYPERTENSIONAHA. 110.164244.

27. Bataller R, Gabele E, Schoonhoven R, Morris T, Lehnert M, Yang L, Brenner DA, Rippe RA. Prolonged infusion of angiotensin ii into normal rats induces stellate cell activation and proinflammatory events in liver. American Journal of Physiology-Gastrointestinal and Liver Physiology. 2003; 285: G642-G651.

28. Wang L, Kamijo Y, Matsumoto A, Nakajima T, Higuchi M, Kannagi R, Kyogashima M, Aoyama T, Hara A. Kidney transplantation recovers the reduction level of serum sulfatide in esrd patients via processes correlated to oxidative stress and platelet count. Glycoconj. J. 2011; 28: 125.

29. Nakajima T, Kamijo Y, Yuzhe H, Kimura T, Tanaka N, Sugiyama E, Nakamura K, Kyogashima M, Hara A, Aoyama T. Peroxisome proliferator-activated receptor $\alpha$ mediates enhancement of gene expression of cerebroside sulfotransferase in several murine organs. Glycoconj. J. 2013; 30: 553-560.

30. Kamijo Y, Hora K, Kono K, Takahashi K, Higuchi M, Ehara T, Kiyosawa K, Shigematsu H, Gonzalez FJ, Aoyama T. Ppara protects proximal tubular cells from acute fatty acid toxicity. J. Am. Soc. Nephrol. 2007; 18: 3089-3100.

31. Spijkers LJ, Janssen BJ, Nelissen J, Meens MJ, Wijesinghe D, Chalfant CE, De Mey JG, Alewijnse AE, Peters SL. Antihypertensive treatment differentially affects vascular sphingolipid biology in spontaneously hypertensive rats. PLoS One. 2011; 6: e29222.

32. Haffner SM, Valdez RA, Hazuda HP, Mitchell BD, Morales PA, Stern MP. Prospective analysis of the insulin-resistance syndrome (syndrome x). Diabetes. 1992; 41: 715-722.

33. Kozono M, Uto H, Ibusuki R, Arima S, Oda K, Taguchi H, Sasaki F, Nasu Y, Hashimoto S, Setoyama H. Antihypertensive therapy improves insulin resistance and serum levels of interleukin- 6 and-10 in 
spontaneously hypertensive rats with steatohepatitis. Mol. Med. Report. 2016; 14: 5385-5394.

34. Reaven GM, Lithell H, Landsberg L. Hypertension and associated metabolic abnormalities - the role of insulin resistance and the sympathoadrenal system. N. Engl. J. Med. 1996; 334: 374-382.

35. Spranger J, Kroke A, Möhlig M, Hoffmann K, Bergmann MM, Ristow M, Boeing H, Pfeiffer AF. Inflammatory cytokines and the risk to develop type 2 diabetes: Results of the prospective population-based european prospective investigation into cancer and nutrition (epic)-potsdam study. Diabetes. 2003; 52: 812-817.

36. Langeveld M, Aerts JM. Glycosphingolipids and insulin resistance. Prog. Lipid Res. 2009; 48: $196-205$.

37. Buschard K, Fredman P, Bøg - Hansen E, Blomqvist M, Hedner J, Råstam L, Lindblad U. Low serum concentration of sulfatide and presence of sulfated lactosylceramid are associated with type 2 diabetes. The skaraborg project. Diabet. Med. 2005; 22: 1190-1198.

38. Bovin LF, Fredman P, Månsson J-E, Buschard K, Bendtzen K. In vitro production of cytokines is influenced by sulfatide and its precursor galactosylceramide. FEBS Lett. 1999; 455: 339-343.

39. Buschard K, Diamant M, Bovin LF, MÅNsson JE, Fredman R, Bendtzen K. Sulphatide and its precursor galactosylceramide influence the production of cytokines in human mononuclear cells. APMIS. 1996; 104: 938-944.

40. Merten M, Beythien C, Gutensohn K, Kühnl P, Meinertz T, Thiagarajan P. Sulfatides activate platelets through p-selectin and enhance platelet and platelet-leukocyte aggregation. Arterioscler. Thromb. Vasc. Biol. 2005; 25: 258-263.

41. Li G, Hu R, Guo Y, He L, Zuo Q, Wang Y. Circulating sulfatide, a novel biomarker for ST-segment elevation myocardial infarction. J Atheroscler. Thromb. 2018: 43976. 


\section{Figure Legends}

Figure 1 Systolic blood pressure alterations in mice modeling hypertension (hypertension), mice receiving low blood pressure treatments (low blood pressure) and mice modeling hypertension that also received treatments (hypertension treatment) are expressed in the top, middle and bottom of the figure, respectively. On Day 0, 10-week-old male C57BL/6j mice were treated with a standard rodent diet or a diet with antihypertensive drugs mixed into it. Meanwhile, osmotic minipumps were implanted to infuse Angll. Systolic blood pressure was measured every 7 days and at each time point, with over 20 measurements recorded and averaged. Con, control; Los, losartan; Irbe, irbesartan; Nif, nifedipine; Hyd, hydralazine; A+Los, Angll+losartan; A+Irbe, Angll+irbesartan; A+Nif, Angll+nifedipine; A+Hyd, Angll+hydralazine. The results are expressed as the mean \pm SEM. (Control group $n=10$, other groups $n=5$ respectively). ${ }^{*} P<0.05$, ${ }^{* *} P<0.001$ vs. Control.

Figure 2 Serum and liver sulfatide levels and compositions from hypertension, low blood pressure and hypertension treatment mice. (A) Serum and liver sulfatide levels were measured by MALDI-TOF MS. The levels of sulfatides were calculated as the sum of 7 lysosulfatides species. HT, hypertension; LBP, low blood pressure; HT-T, hypertension treatment. (B) Compositions of 7 lysosulfatides species from serum and liver. d18:2, LS-sphingadienine; d18:1, LS-(4E)-sphinganine; d18:0, LS-sphinganine; t18:0, LS-phytosphingosine; d20:1, LS-(4E)-icosasphingenine; d20:0, LS-icosasphingenine; t20:0, LS-4D-hydroxyicosasphingenine. The results are expressed as the mean \pm SEM. (Control group $n=10$, other groups $n=5$ respectively). ${ }^{*} P<0.001$ vs. Control, ${ }^{\#} P<0.001$ vs. Angll high dose. Open bars: no Angll infusion, striped bar: low dose Angll infusion, closed bars: high dose Angll infusion. 
Figure 3 Expression of enzymes involved in sulfatide biosynthesis, degradation and transport in livers from hypertension, low blood pressure and hypertension treatment mice. (A) Expression of mRNAs encoding Cst, Cgt, Sptlc2, Arsa, Galc and Gltp. mRNA levels are normalized to Gapdh mRNA levels and are shown as fold changes relative to that observed in mice from the Control group. (B) Immunoblot analysis of CST. Thirty micrograms of whole-liver lysate protein prepared from each mouse was loaded into each well for electrophoresis. Actin was used as the loading control. The band intensity was measured densitometrically, normalized to those of actin and shown as values relative to that of mice from Control group. The results are expressed as the mean \pm SEM. (Control group $n=10$, other groups $n=5$ respectively). $* P<0.05$, ${ }^{* *} P<0.001$ vs. Control, ${ }^{\#} P<0.05,{ }^{\# \#} P<0.001$ vs. Angll high dose. Open bars: no Angll infusion, striped bar: low dose Angll infusion, closed bars: high dose Angll infusion.

Figure 4 Expression of enzymes involved in reactive oxygen species metabolism and the tissue oxidation stress marker MDA in livers from hypertension, low blood pressure and hypertension treatment mice. (A) Hepatic levels of MDA. (B) Expression of mRNAs for Cat, Sod1, Gpx1, Nox2, Ncf1 and Acox1. mRNA levels are normalized to Gapdh mRNA levels and shown as fold changes relative to that observed in mice from the Control group. (C) Immunoblot analysis of CAT, NOX2, ACOX1. Thirty micrograms of whole-liver lysate protein prepared from each mouse was loaded into each well for electrophoresis. Actin was used as the loading control. The band intensity was measured densitometrically, normalized to those of actin and shown as values relative to that of mice in Control group. The results are expressed as the mean \pm SEM. (Control group $n=10$, other groups $n=5$ respectively). ${ }^{*} P<0.05, * * P<0.001$ vs. Control, ${ }^{\# \#} P<0.001$ vs. Angll high dose. Open bars: no Angll infusion, striped bar: low dose Angll infusion, closed bars: high dose Angll infusion. 
Figure 5 Expression of PPAR $\alpha$ and its target genes LACS and PT in livers from hypertension, low blood pressure and hypertension treatment mice. (A) Expression of mRNAs encoding Ppara, Lacs and Pt. mRNA levels are normalized to Gapdh mRNA levels and shown as fold changes relative to that observed in mice from the Control group. (B) Immunoblot analysis of PPAR $\alpha$, LACS and PT. Thirty micrograms of the nuclear fraction or whole-liver lysate protein prepared from each mouse was loaded into each well for electrophoresis. TBP or Actin was used as the loading control respectively. The band intensity was measured densitometrically, normalized to those of TBP or actin and shown as values relative to that observed in mice from the Control group. The results are expressed as the mean \pm SEM. (Control group $n=10$, other groups $n=5$ respectively) ${ }^{*} P<0.05,{ }^{*} P<0.001$ vs. Control, ${ }^{\#} P<0.05,{ }^{\#} P<0.001$ vs. Angll high dose.

Figure 6 Scheme relating the complex effects of sulfatide metabolism in livers from hypertension, low blood pressure and hypertension treatment mice. Angll infusion induced both hypertension and activation of the renin angiotensin system (RAS) in mice. Consequently, these caused reduction of hepatic CST expression, and decreased serum sulfatide levels as shown. All four antihypertensive drugs can alleviate the abnormally reduced serum sulfatide by reducing hypertension. Losartan and irbesartan antagonize RAS activation through an ARB-specific mechanism. This effect of ARBs ameliorated the enhancement of OS, which contributed to improving serum sulfatide levels. The exclusive effect of PPAR $\alpha$ activation by irbesartan led to increased CST expression and ameliorated levels of OS. These effects by irbesartan restored the greatest levels of serum sulfatide between the four antihypertensive drugs. 
Figure 1
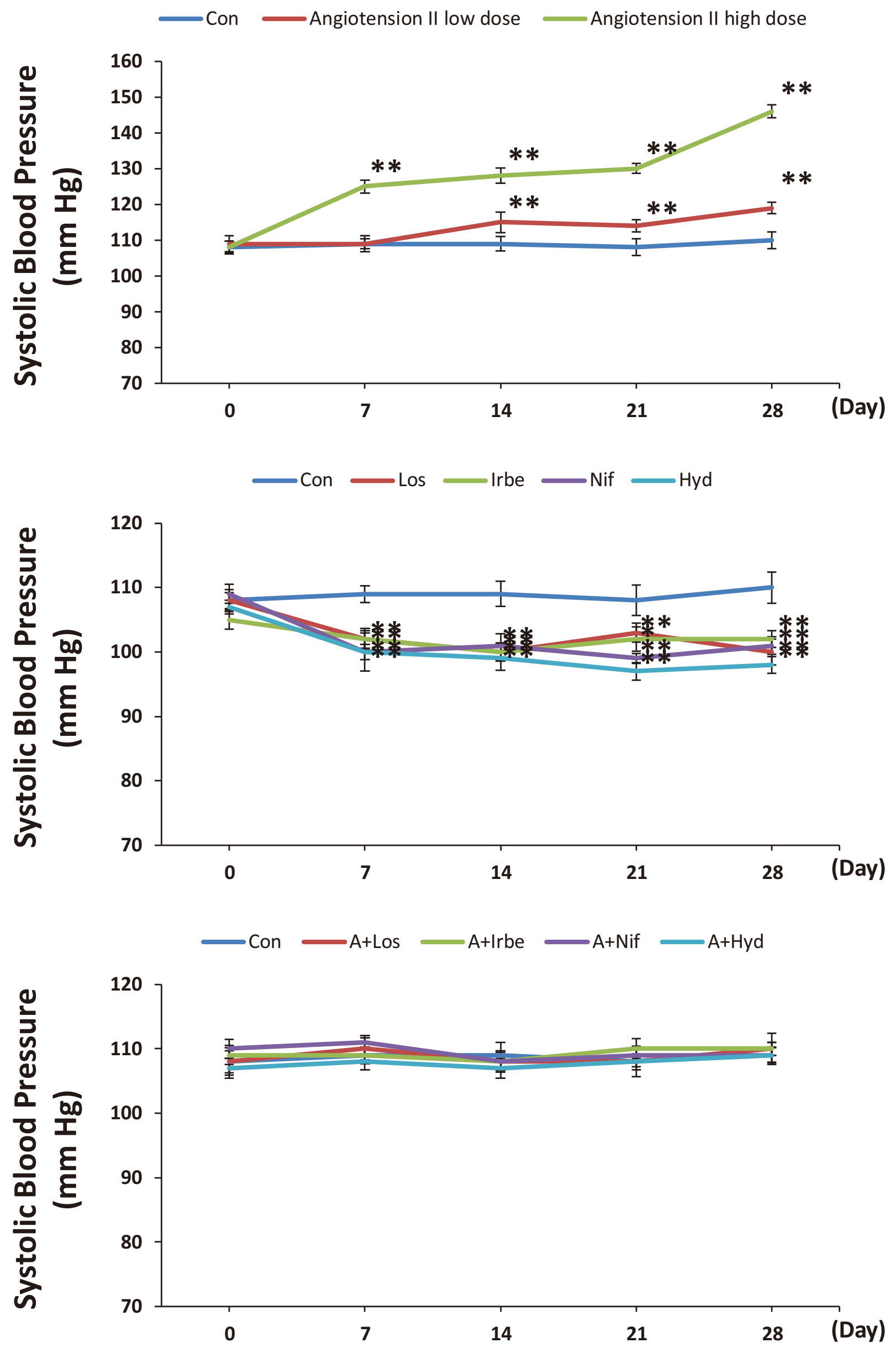
Figure 2

A

Serum sulfatide $(\mathrm{nmol} / \mathrm{ml})$

Liver sulfatide (nmol/g)
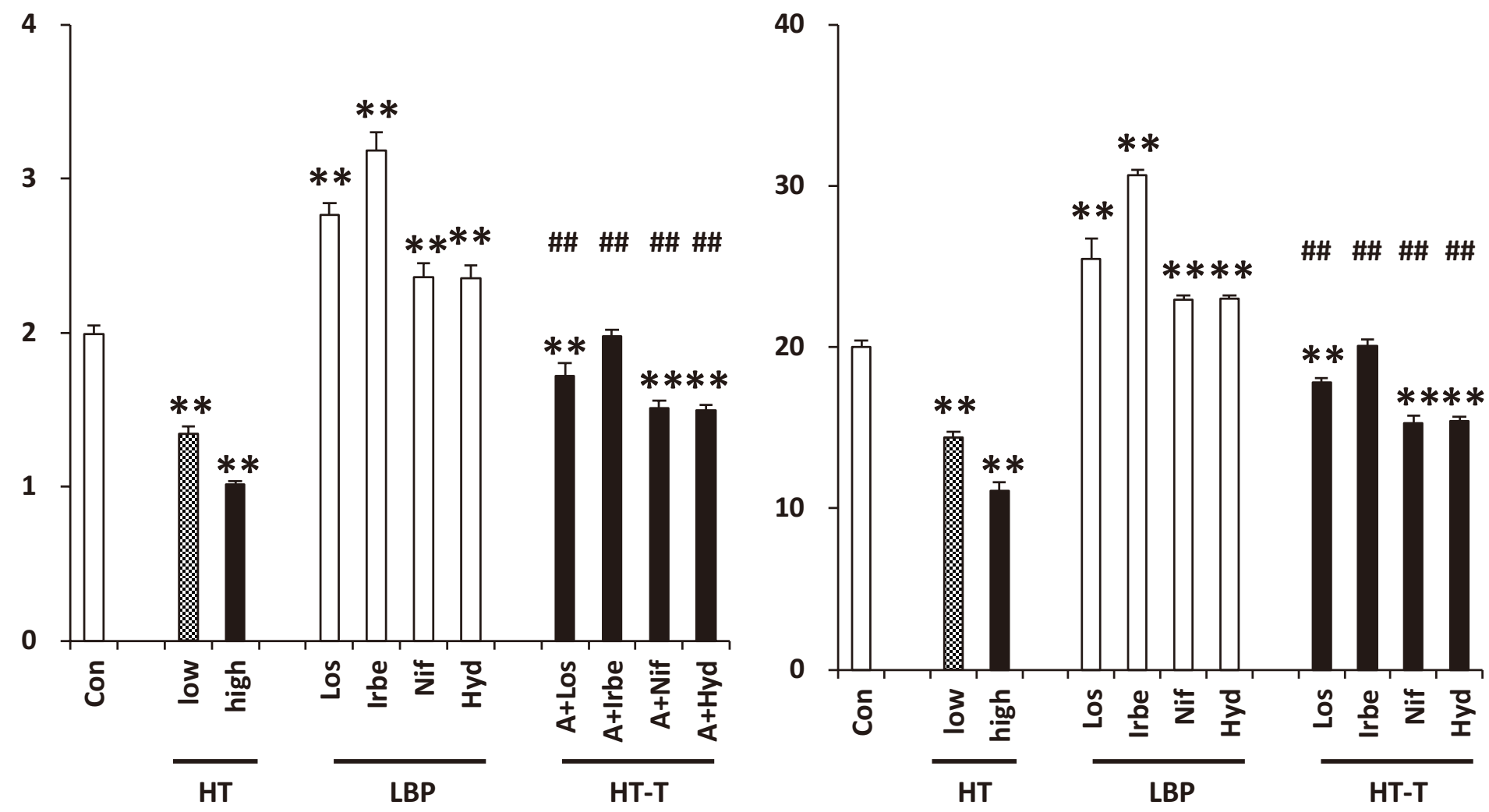

B

Compositions of serum sulfatides

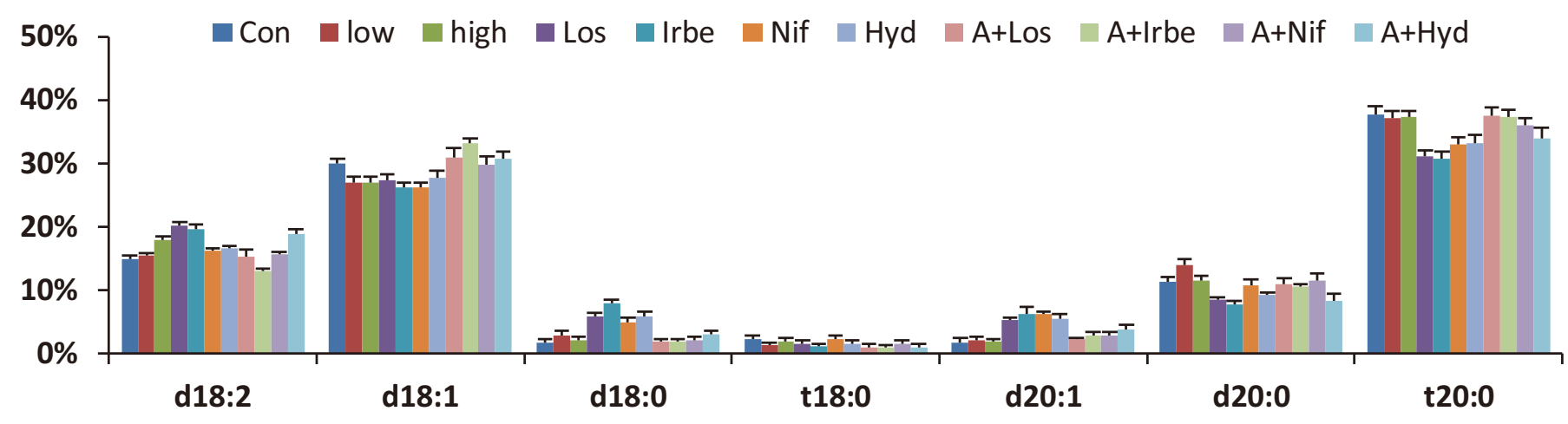

Compositions of liver sulfatides

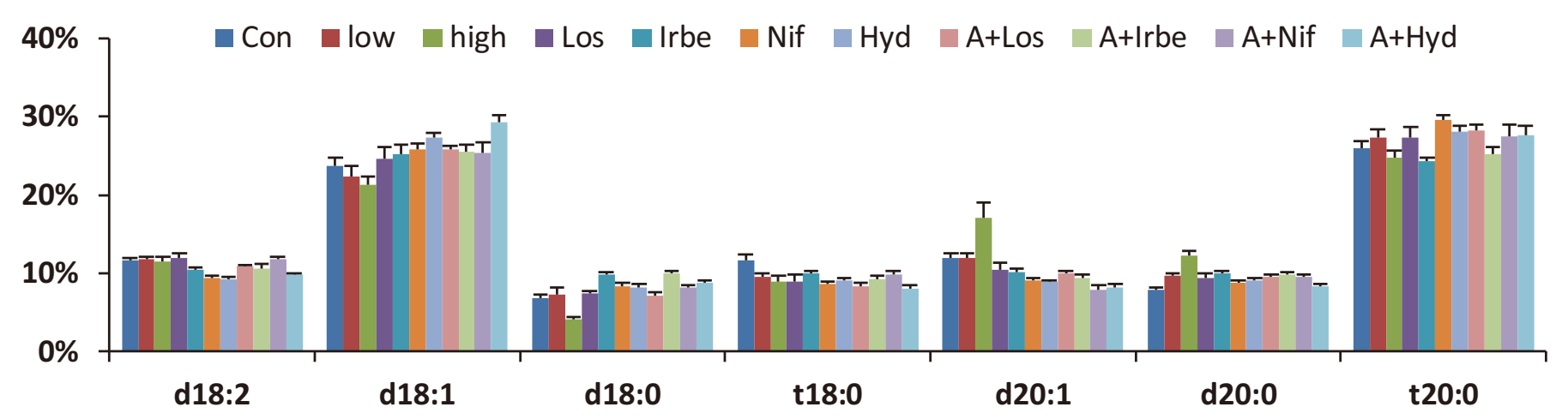


Figure 3

A
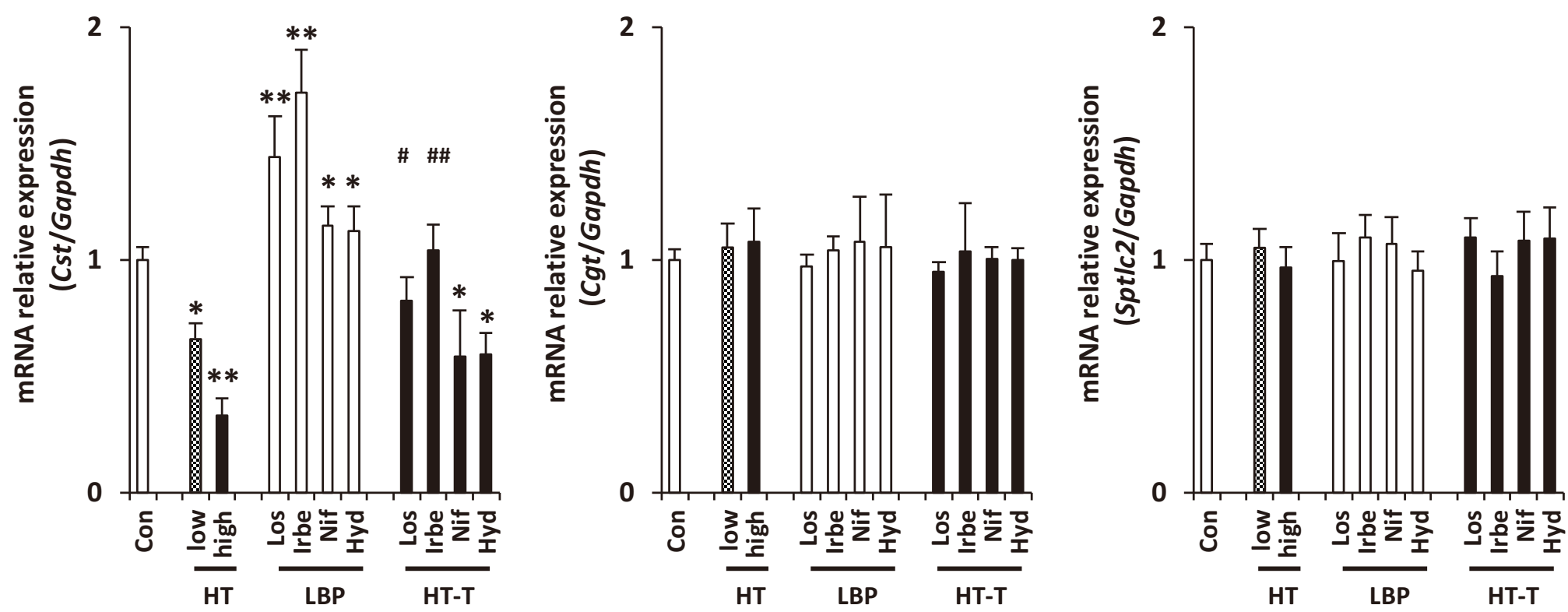

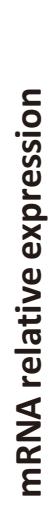
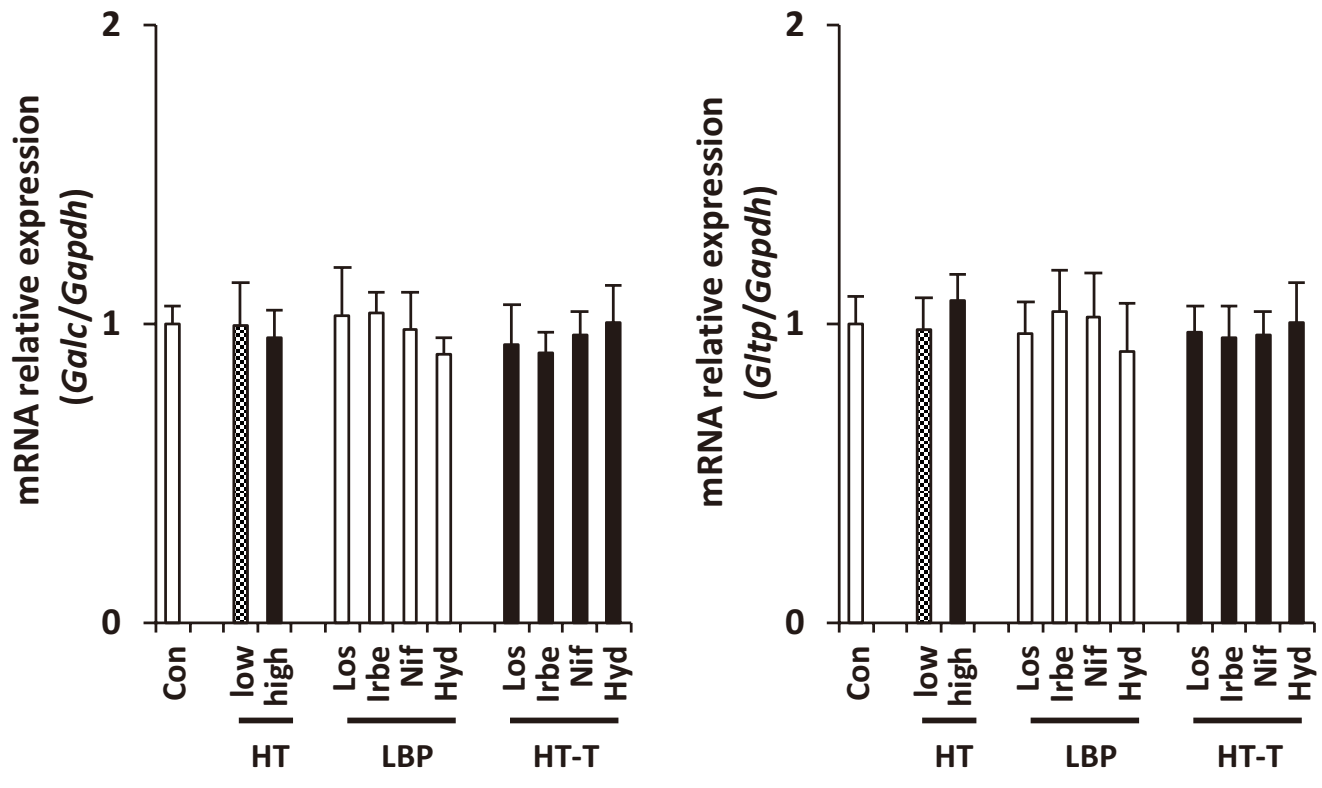

B

CST $\rightarrow$

(47kDa)

Actin $\rightarrow$

(42kDa)
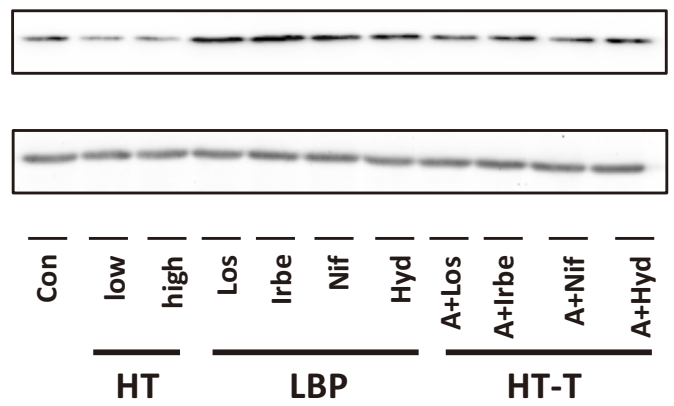

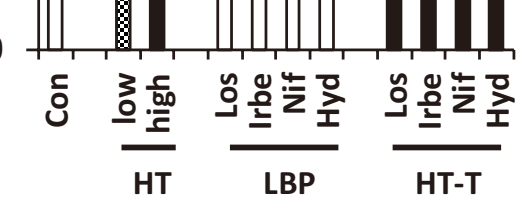

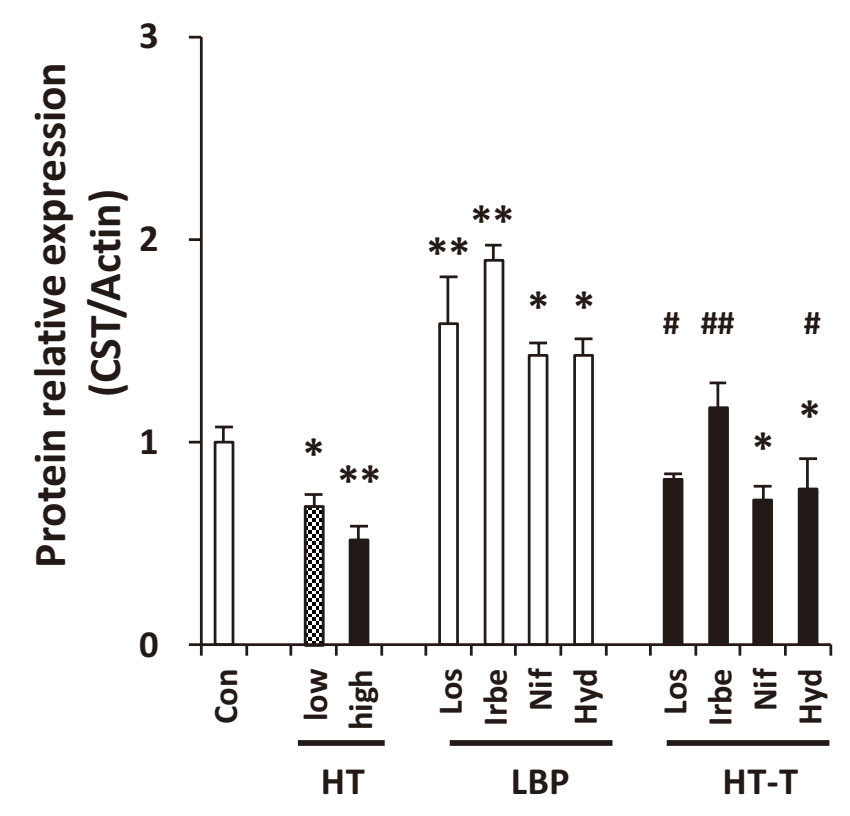

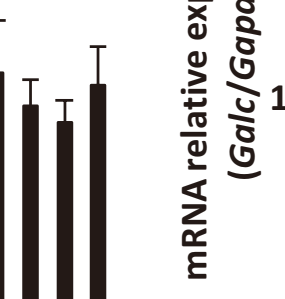


Figure 6

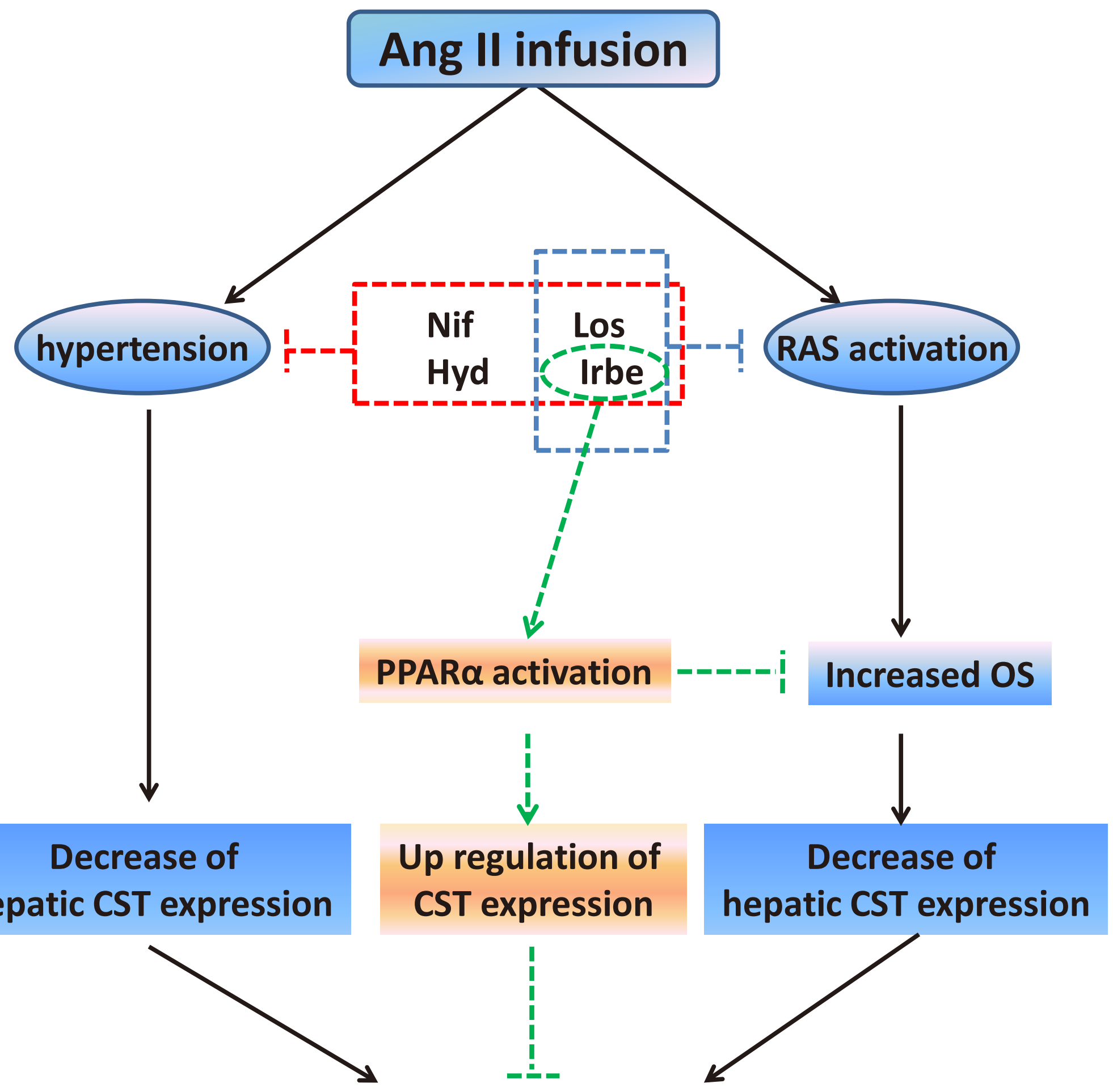

Decrease of serum sulfatides content 